\title{
NUEVA POBLACIÓN DE DRYMAEUS MOUSSONI (MOLLUSCA: BULIMULIDAE) EN LA HISPANIOLA
}

\begin{abstract}
Altagracia Espinosa
Instituto de Investigaciones Botánicas y Zoológicas Prof. Rafael M. Moscoso.Universidad Autónoma de Santo Domingo, Santo Domingo, República Dominicana. altagraciaespinosa@yahoo.com
\end{abstract}

\section{RESUMEN}

Después de tres décadas de ser observado en la República de Haití, se localizan poblaciones de Drymaeus moussoni (L. Pfeiffer, 1853) en la República Dominicana, siendo ésta la primera vez que se registra para una localidad exacta en este último país.

Palabras clave: molusco, Bulimulidae, La Hispaniola.

Title: New population of Drymaeus moussoni (Mollusca: Bulimulidae) on Hispaniola Island.

\section{ABSTRACT}

After three decades of being reported for the Republic of Haiti, populations of Drymaeus moussoni (L.Pfeiffer, 1853) were found in the Dominican Republic, being reported for the first time on that side of the island at a specific locality.

Key words: snails, Bulimulidae, Hispaniola Island.

El molusco terrestre Drymaeus moussoni (L. Pfeiffer, 1853) pertenece a la familia Bulimulidae, es un molusco terrestre endémico de La Hispaniola, cuya concha generalmente es ovalada-cónica a cilíndrica (Breure, 1974). En la Hispaniola, esta familia está representada por los géneros: Bulimulus Leach, 1814 y Drymaeus Albers, 1850. El género Drymaeus se caracteriza por la presencia en su protoconcha de cordones axiales y espirales que se entrecruzan formando ángulos de 90 grado. En su descripción original, Drymaeus moussoni fue registrado para la localidad "Santo Domingo" (Pfeiffer, 1853) no precisándose exactamente su ubicación. Sesenta y cuatro años después, en 1917, fue colectado por C. J. Hunn en la localidad Debajo Pont Sonde, República de Haití. El Dr. Fred Thompson (Curator of Non-marine Malacology, Florida Museum of Natural History, University of Florida) en un viaje a la isla, lo colecta en el 1979 en las localidades de Las Caobas, Dufailly y Gonaives ubicadas en la República de Haití.

En el mes de febrero de 2010, los investigadores Ruth Bastardo, Manuel Valdez y Altagracia Espinosa del Instituto de investigaciones Botánicas y Zoológicas (IIBZ) y la maestra Maricela Tejada, de la escuela de Biología de la Universidad Autónoma de Santo Domingo, visitaron la comunidad de Bánica y observaron la población de esta especie. Anteriormente, esta población fue fotografiada por Miguel Ángel Landestoy, quien nos acompañó en el trabajo de campo. Una vez en el laboratorio, se tomaron las medidas de las conchas usando un vernier digital modelo DC-122a , registrándose alturas de concha desde 18.60 a 27.97 mm y diámetro desde 9.46 a 13.56 $\mathrm{mm}$.

Esta localidad se corresponde con Bosque seco, de acuerdo a Häger y Zanoni (1993). En un área de $10 \times 10 \mathrm{~m}$, con impacto humano, se encontraron 48 individuos de D. moussoni (4.8 indv $/ \mathrm{m}^{2}$ ), observándose estos en los troncos de Haematoxylon campechianum L. (campeche), Crescentia cujete L. (higüero) y Guaiacum officinale L. (guayacán). Los individuos de $D$. moussoni se encontraron adheridos a los troncos por un fuerte epifragma y se observaron tanto 

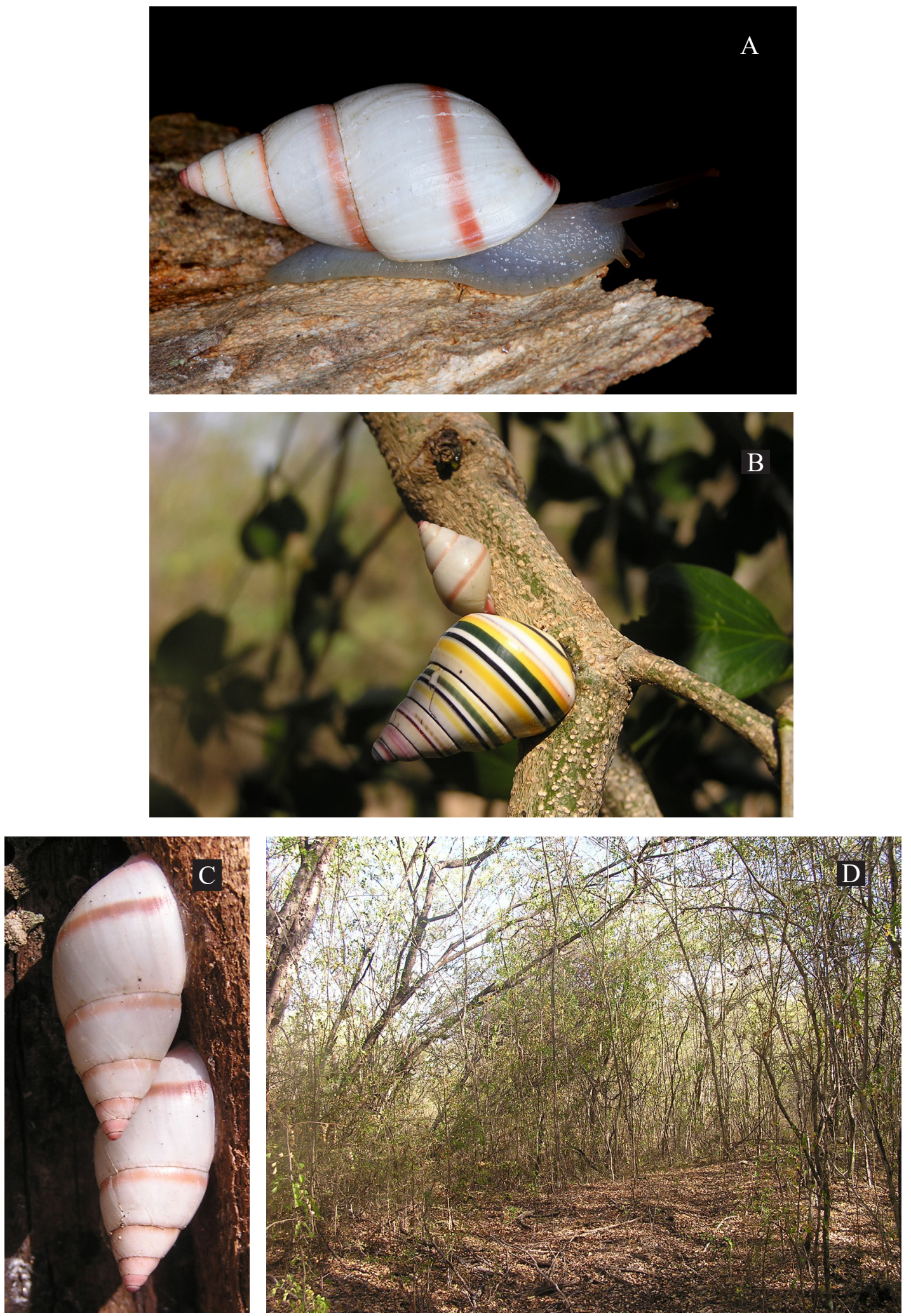

Figura 1.Vistas de Drymaeus moussoni y su hábitat. A, en actividad. B, asociado a Liguus virgineus. C, en estivación. D, hábitat en Bánica, República Dominicana. 


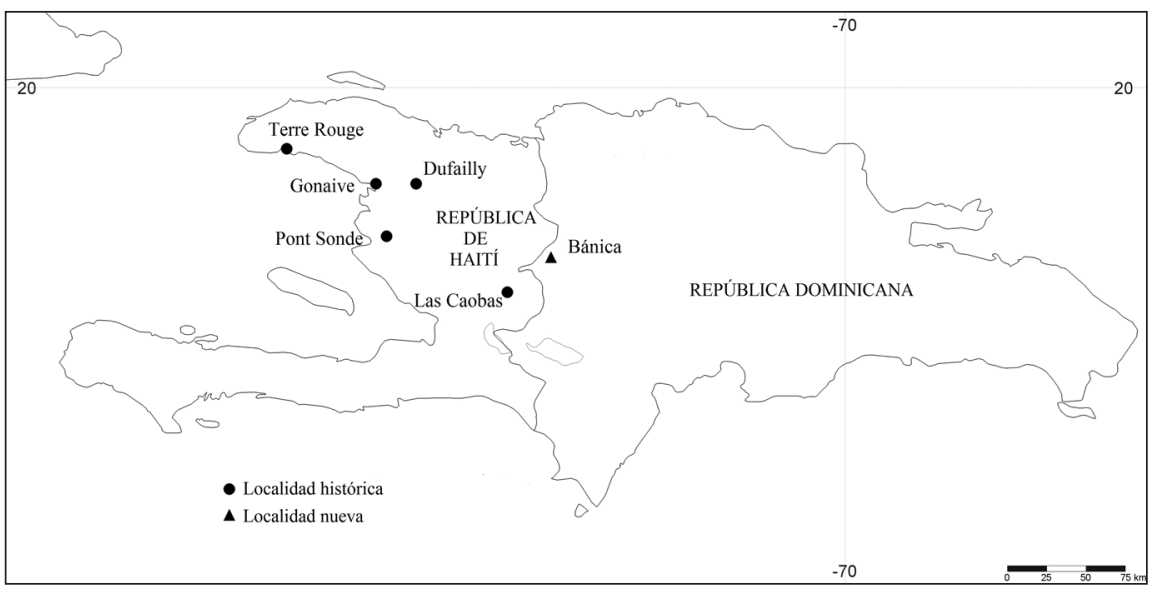

Figura 2. Distribución de Drymaeus moussoni en La Hispaniola.

de forma individual como en grupos de tres (3) y de seis (6). No se observó a $D$. moussoni activo en la vegetación. La única especie de molusco que se encontró asociado a $D$. moussoni fue Liguus virgineus (L.). Los ejemplares están depositados en la colección del IIBZ.

Localidades. REPÚBLICA DE HAITÍ: Debajo de Pont Sonde, Artibonite Riv. Bertrand Plantation, C. J. Hunn, Aug/1917. No. de catálogo: Malacology 143779. Disponible en www. mczbase.mcz.harvard.edu. Nr. Croix Malat, Artibonite Plain, C. J. Hunn, Aug/1917. No. de catálogo: Malacology 143780. Disponible en www.mczbase.mcz.harvard.edu. Terre Rouge [sin otros datos], No. de catálogo: Malacology 103926. Disponible en www.mczbase.mcz.harvard. edu. 2 Km SE of Las Cahobas, Dept. de 1' Quest, F. G. Thompson, 4/16/ 1979, No. de catálogo: 32617. Disponible en WWW.flmnh.ufl.edu. 9 km NE of Dufailly. Dept. de 1' Quest, colector : F. G. Thompson. 5/1/1979. No. de catálogo: 32872. Disponible en www. Flmnh.ufl.edu. $1 \mathrm{~km} \mathrm{NE}$ of Dufailly. Dept. de 1' Quest, F. G. Thompson. 5/1/1979, No. de catálogo 32894. Disponible en www. Flmnh.ufl.edu. Near Gonaives. República Haiti. Colector : J.J. Brown. No de catálogo : 176955. Disponible en www. Flmnh.ufl.edu. REPÚBLICA DOMINICANA: Los Yareyes. 2.3 Km de la entrada de Bánica, Elías Piña, M. A. Landestoy, R. Bastardo, M. Valdez, M. Tejada y A. Espinosa, 13/feb/2010, 19 Q 0217292 E 2106694 N, Alt. 327 msnm.

\section{AGRADECIMIENTOS}

A Miguel Ángel Landestoy por su colaboración con las actividades que desarrolla el IIBZ y el permiso para la utilización de su fotografía. A la maestra Ruth Bastardo, directora del IIBZ por su apoyo en todo el proceso y por las fotografías. A los maestros Manuel Valdez y Maricela Tejada por su ayuda en las actividades de campo.

\section{LITERATURA CITADA}

Breure, A. 1974. Caribbean Land Molluscs: Bulimulidae. I. Bulimulus. Studies on the Fauna of Curacao and other Caribbean Islands, (145): 1-80.

Häger, J. y T.A. Zanoni.1993. La vegetación natural de la República Dominicana: Una nueva clasificación. Moscosoa 7: 39-81

Pfeiffer, L. 1853. Description of new Land Shells from the Collection of H. Cuming. Proceeding of the Zoological Society of London. (for 1851) 19,:147-148.

[Recibido: 12 de abril, 2012. Aceptado para publicación: 18 de mayo, 2012] 